\title{
Macro- and microscale structure formation and aging in different arrested states of Laponite dispersions
}

\author{
M. Pilavtepe, ${ }^{1, a)}$ S. M. Recktenwald, ${ }^{1}$ R. Schuhmann, ${ }^{2}$ K. Emmerich, ${ }^{2}$ and N. Willenbacher ${ }^{1}$ \\ ${ }^{1}$ Institute for Mechanical Process Engineering and Mechanics, Karlsruhe Institute of Technology, 76131 Karlsruhe, \\ Germany
}

${ }^{2}$ Competence Center for Material Moisture, Karlsruhe Institute of Technology, 76344 Karlsruhe, Germany

\begin{abstract}
Structure formation and aging in different arrested states of Laponite dispersions have been investigated at the macro and microscale. Covering a wide range of solid content and salt concentrations at different $p \mathrm{H}$ glasses, strong and weak gels with prevailing edge to face (EF) or face to face (FF) layer contacts were formed. Mechanical shear and squeeze flow rheometry were combined with diffusing wave spectroscopy and multiple particle tracking (MPT) microrheology. Strong attractive gels form much more quickly than weak gels, and particularly repulsive glasses. Gels with preferred EF contacts are stronger and are created more quickly than gels with prevailing FF contacts. Strong gels show little aging and exhibit a weak increase of $G^{\prime} \sim t^{\alpha}$ with $\alpha=0.11 \pm 0.03$, higher $\alpha$ values are found for weak gels, and the strongest aging is observed in glasses. MPT data reveal structural refinement at the submicrometer length scale during aging for gels but not for glasses. Strong structural heterogeneity most pronounced at $p \mathrm{H}=8.5$ occurs during gel or glass formation, but at longer times, all arrested states appear homogenous at the $0.2 \mu \mathrm{m}$ length scale. Finally, all arrested states exhibit power law frequency dependence $G^{\prime \prime} \sim \omega^{0.75}$ at high frequencies attributed to internal bending modes of layers.
\end{abstract}

\section{INTRODUCTION}

The phase behavior, structure and dynamics of clay mineral colloidal dispersions are of high technical relevance and great scientific interest. Engineering challenges include soil mechanics, application of construction materials or processing of pastes including clay mineral particles as additives. The variety of different dynamically arrested states observed in such systems has stimulated the interest of soft matter scientists. Fundamental investigations address phase behavior and particularly structure and dynamics of different arrested states [ $\left[\begin{array}{ll}1 & 7\end{array}\right]$. These features are controlled by the strength and range of electrostatic interactions among particles strongly varying with $p \mathrm{H}$ and added electrolyte as schematically sketched in Fig. 1. Laponite dispersions have been investigated as a model system for clay mineral suspensions [8] and disklike colloids [9 11 ] in general. Laponite is a synthetic hectorite, and the platelike particles carry permanent negative charges due to isomorphic substitution. Edges are positively charged at low $p \mathrm{H}$, but negative edge charges occur at high $p \mathrm{H}$. The point of zero charge at edges,

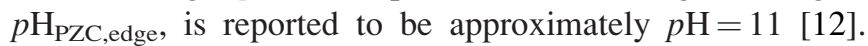
Even if the inherent $p \mathrm{H}$ of Laponite dispersion is lower than its $p \mathrm{H}_{\mathrm{PZC} \text {,edge }}$, in the absence of ions or at very low ionic strength, the extended electrical double layer around the

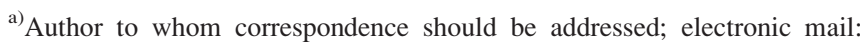
muege.pilavtepe@kit.edu
}

dispersed layers leads to the formation of an arrested glass structure $[1,3,13,14]$. Setting $p \mathrm{H}$ or adding an electrolyte leads to a contraction of the electrical double layer, allowing for direct layer contacts, and so-called attractive arrested states, also termed gels, are formed [1,3]. At

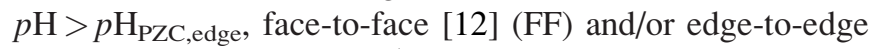
[15] (EE) contacts with $\mathrm{Na}^{+}$in between are formed, resulting in a so-called partially parallel overlapped (PPO) gel structure $[16,17]$. At high $p \mathrm{H}$ and high electrolyte concentration, the possibility of FF layer orientation is higher than EE inter-

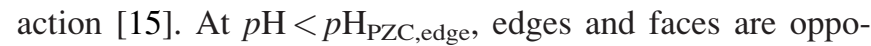
sitely charged, and edge-to-face (EF) contacts occur, which are supposed to result in arrested gel states with a so-called house of cards (HOC) structure [18 21]. Models for structure formation in clay mineral dispersions are often based on these assumptions about different types of particle contacts mentioned above. The existence of so-called PPO or HOC structures is widely accepted in the clay mineral science community $[12,14,15,18,22$ 25]. However, there is no direct experimental evidence for these particle contacts except for Monte Carlo simulations [19] regarding gelation in clay mineral suspensions considering electrostatic interactions among the platelike particles and cryo-TEM 3D-images of smectite particles in aqueous environment [24]. The physical motivation for these structural models is the experimental fact that the rim or edge charge changes from positive at $p \mathrm{H}<p \mathrm{H}_{\mathrm{PZC} \text {,edge }}$ to negative at higher $p \mathrm{H}$. The edge charge of Laponite has been quantitatively determined using potentiometric and mass titration techniques [26]. 


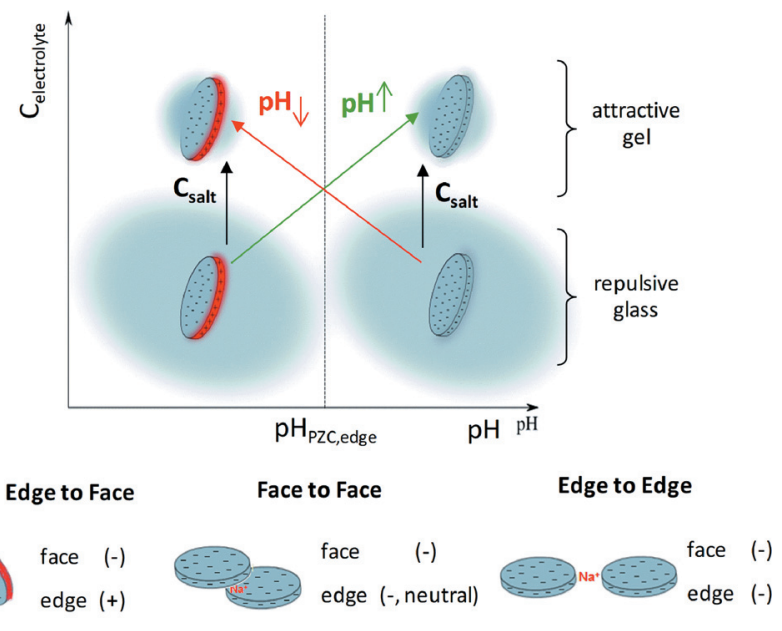

FIG. 1. Effect of electrolyte concentration and $p \mathrm{H}$ on surface charge and range of electrical double layer in aqueous Laponite dispersions. Negative charges are marked in blue, positive charges in red. The different pathways of changing surface charge and electrostatic interaction are marked by arrows.

Structure formation and aging in clay mineral dispersions strongly depend on particle and salt concentration [7,27]. Due to the short-range attractive forces among Laponite layers, gel structures form more quickly than glass structures built by long-range repulsive forces [3].

The kinetics of the gel/glass formation in Laponite dispersions at different solids content and electrolyte concentration has been studied by numerous authors covering time intervals from hours to years using light and $\mathrm{X}$-ray scattering [5,27 31] as well as rheological methods [13,32 37]. Willenbacher [36] found a power law relationship between complex viscosity and time $\left(\left|\eta^{*}\right| \sim t^{0.13 \pm 0.02}\right)$ for gel-like Laponite dispersions holding within the time regime up to $10^{6} \mathrm{~s}$. As noted by Ruzicka and Zaccarelli [6], a deficiency of some other early studies on Laponite phase behavior is the neglect of sample aging $[4,38]$, which may have led to inaccurate interpretation of arrested states of dispersions. Therefore, many different phase diagrams of Laponite dispersions are available $[3,4,7,29,39]$. Hence, a clear picture of layer interaction at different $p \mathrm{H}$, electrolyte concentration, and the influence of electrolyte concentration on the kinetics of phase transition of Laponite dispersion is still lacking.

Laponite dispersions with low solid concentration and without added salt need more time to reach an arrested state, and cluster size changes during this time. Therefore, such dispersions are called heterogeneous gels at a macroscopic length scale [28]. At higher clay mineral concentration, the arrested state forms more quickly, and there is no evidence for structural heterogeneity from dynamic light or small X-ray scattering; accordingly, such states are called homogeneous gel or attractive glass $[28,30]$. On the other side, microrheological studies using particle tracking methods revealed that heterogeneous gel-like structures occur in Laponite dispersions at low solids and high electrolyte concentration [32,40], and homogeneous repulsive glass structures were observed in the absence of electrolyte [33]. Oppong et al. [32] observed an increase of heterogeneity during structure formation until the characteristic crossover time at which $G^{\prime}=G^{\prime \prime}$ was reached with no further change beyond that time. Their results, however, may be obscured by aging effects as they added the tracer particles one month after sample preparation, assuming that complete rejuvenation is possible by vigorous sonication. Rich et al. [40] observed a gradual shift of sol-gel transition in their particle tracking experiments depending on the size of the used tracer particles and found an increase in heterogeneity up to the crossover time but did not continue to monitor this at longer times. Jabbari-Farouji et al. [33] claimed that glass Laponite dispersions are homogeneous based on microrheological experiments using probe particles with $1.16 \mu \mathrm{m}$ diameter but observed heterogeneity in the gel they investigated using $0.5 \mu \mathrm{m}$ probe particles. However, this optical tweezer-based study suffers from poor statistical significance, since the viscoelastic response of the gel sample was probed only at 58 different positions.

Here, we have used classical oscillatory shear rheometry and multiple particle tracking optical (MPT) microrheology to characterize kinetics of structure formation and aging for different types of repulsive or attractive arrested states. We have investigated Laponite dispersions with a broad range of clay mineral content ( $13 \mathrm{wt}$. \%), added salt concentration (up to $10{ }^{2} \mathrm{M} \mathrm{NaCl}$ ) and different $p \mathrm{H}(8.5,10$, and 12). The variety of methods applied in this study and the broad range of physicochemical parameters investigated provide a comprehensive scientific view of differences between arrested states with different Laponite layer orientations. A distinction between repulsive glasses and attractive gels from bulk rheological measurements alone is not trivial and sometimes may be impossible [41]. Therefore, we have combined bulk rheology with microrheology to shed new light on the longstanding controversy about the rheological behavior of Laponite dispersions at the macro- and microlength scales. We discuss the effect of the main control parameters i.e., clay mineral content and salt concentration, as well as $p \mathrm{H}$ on kinetics of structure formation, aging, mechanical strength, microscopic heterogeneity and structural refinement for different attractive and repulsive arrested states. Beyond that, we have employed squeeze flow mechanical rheometry and diffusing wave spectroscopy (DWS) optical microrheology to determine linear viscoelastic properties in the frequency range up to $10^{6} \mathrm{rad} / \mathrm{s}$. We have investigated Laponite dispersions at various $p \mathrm{H}$ and electrolyte concentrations.

\section{MATERIALS AND METHOD}

\section{A. Materials}

The synthetic hectorite Laponite RD (registered trademark of BYK Additives) was donated by BYK Additives \& Instruments, Germany. Its general formula is $\mathrm{Na}_{0.38} \mathrm{Si}_{8}\left(\mathrm{Mg}_{5.72} \mathrm{Li}_{0.18}\right) \mathrm{O}_{20}(\mathrm{OH})_{4}$. Laponite RD has a specific density of $2700 \mathrm{~kg} / \mathrm{m}^{3}$, specific surface area of $370 \mathrm{~m}^{2} /$ $\mathrm{g}$, and cation exchange capacity (CEC) of $79 \mathrm{cmol}(+) / \mathrm{kg}$ determined according to Meier and Kahr [42] and Delavernhe et al. [43]. Laponite consists of a delaminated state of nearly monodisperse, rigid, disk-shaped layers with a thickness slightly less than $1 \mathrm{~nm}$ and a diameter of approximately $30 \mathrm{~nm}[38,39]$. 


\section{B. Sample preparation}

The effect of solids content and added salt concentration on the flow behavior of Laponite suspensions was studied in the range of $13 \mathrm{wt} . \%$ and $010^{2} \mathrm{M} \mathrm{NaCl}$. The corresponding amount of Laponite powder was dispersed under agitation in the corresponding ionic aqueous solution. Samples were homogenized in an ultrasonic bath for $20 \mathrm{~min}$. Subsequently, samples were treated in an ultrasonic homogenizer (Digital Sonifier, Branson, USA) for $20 \mathrm{~s}$ at $10 \%$ amplitude to ensure delamination and homogeneous distribution of clay mineral layers. During preparation, the sample temperature was kept constant at $25^{\circ} \mathrm{C}$, since heating can influence the aging behavior [44]. We measured the inherent $p \mathrm{H}$ value of Laponite dispersions as $10 \pm 0.2$. The $p \mathrm{H}$ value was set to $p \mathrm{H}=12$ and $p \mathrm{H}=8.5$ by adding $1 \mathrm{M} \mathrm{NaOH}$ or $\mathrm{HCl}$ aqueous solution to the initial dispersion at $p \mathrm{H}=10$. The minimal $p \mathrm{H}$ was set to 8.5 since at lower $p \mathrm{H}$, Laponite starts to dissolve. The rate of this reaction decreases with increasing $p \mathrm{H}$ and is negligible for $p \mathrm{H} \geq 8.5$ [5]. Samples with high salt concentration, approximately $10^{2} \mathrm{M}$, became turbid after setting the $p \mathrm{H}$ value to 8.5 . Such unstable samples were excluded from this study. No turbidity was observed for samples at $p \mathrm{H}=12$. We checked $p \mathrm{H}$ values of all samples for a period of four months, and in accordance with Mongondry et al. [5], we observed that $p \mathrm{H}$ remained constant. The samples were individually prepared for each set of experiments using the same procedure. The electrolyte concentration of dispersion increases upon setting $p \mathrm{H}$ and additionally by released exchangeable cations from the Laponite structure. The $p \mathrm{H}$-dependent CEC of Laponite corresponding to the layer charge was characterized in a wide range of $p \mathrm{H}$ by Delavernhe et al. [45]. The thickness of the electrical double layer (nm) can be estimated via Eq. (1) from the equivalent $\mathrm{NaCl}$ concentration of samples [46] calculated from conductivity of dispersions (see Table I)

$$
\kappa^{1}=\frac{0.304}{\sqrt{ } C_{N a C l, e q}} .
$$

The composition, physicochemical properties, and the type of arrested state of all investigated samples are listed in Table I. This table also gives an overview in which figure results for each sample can be found.

\section{Transient oscillatory shear at fixed frequency and amplitude}

A controlled stress rheometer (HAAKE Rheostress RS 150, Thermo HAAKE, Germany) equipped with a Searle system that consists of a concentric cylinder system with rotating inner cylinder radius $R_{I}=19.36 \mathrm{~mm}$ and outer cylinder radius $R_{2}=20 \mathrm{~mm}$ was used to measure the structure formation and aging dynamics of Laponite dispersions. Directly after sample preparation in sol-state, $10 \mathrm{ml}$ dispersions were filled into the Searle system and sheared at sufficiently high shear stress $\sigma$ (greater than the apparent yield stress $\sigma_{y}$ ) for a given time interval $\Delta t=300 \mathrm{~s}$ that guarantees complete destruction of the possible sample structure. Subsequent recovery of the remaining structure was monitored using small-amplitude oscillatory shear measurements at a stress amplitude of $\sigma_{0}=0.2 \mathrm{~Pa}$ and a frequency of $\omega=0.6 \mathrm{rad} / \mathrm{s}$. The top of the sample chamber was covered with a plate to suppress evaporation of water.

\section{Amplitude sweep experiments}

We applied amplitude sweep experiments at time intervals of one week for a total lag time of up to 16 weeks to characterize the long-term aging and change of mechanical strength of Laponite dispersions. These experiments were carried out using a Physica Anton Paar MCR501 controlled stress rheometer with cone-plate geometry (diameter $25 \mathrm{~mm}$, cone angle

TABLE I. Properties of investigated Laponite dispersions. The supposed structure type of dispersions (EF or FF) is shown in brackets together with the disper sion state (glass, weak or strong gel). The last column shows the numbers of the figures in which data for the respective sample are included.

\begin{tabular}{|c|c|c|c|c|c|c|}
\hline Solids content (wt. \%) & Added $\mathrm{NaCl}$ conc. $(\mathrm{M})$ & $p \mathrm{H}$ & State of dispersion & Conductivity $\left(\mu \mathrm{S} \mathrm{cm}{ }^{1}\right)$ & Calculated electrolyte conc. (M) & Shown in Figure \\
\hline 1 & $10^{2}$ & 10 & Strong gel (EF) & 1368 & $1.4 \times 10^{2}$ & $3(b), 5,9(b)$ \\
\hline 1 & $10^{2}$ & 12 & Weak gel (FF) & 2783 & $3.0 \times 10^{2}$ & 3(b) \\
\hline 2 & 0 & 10 & Repulsive glass & 505 & $3.1 \times 10^{3}$ & 2(a), 2(b), 3(a), 5, 6, 9(a) \\
\hline 2 & 0 & 8.5 & Strong gel (EF) & 892 & $7.8 \times 10^{3}$ & $3(a), 6$ \\
\hline 2 & 0 & 12 & Weak gel (FF) & 1869 & $1.9 \times 10^{2}$ & $3(\mathrm{a}), 6$ \\
\hline 2 & $10^{2}$ & 10 & Strong gel (EF) & 1651 & $1.7 \times 10^{2}$ & 2(a), 2(b), 5,9(b) \\
\hline 2 & $10^{2}$ & 12 & Strong gel (FF) & 3066 & $3.4 \times 10^{2}$ & 5 \\
\hline 2 & $10^{3}$ & 10 & Repulsive glass & 743 & $6 \times 10^{3}$ & 2(b), 6 \\
\hline 2 & $10^{3}$ & 8.5 & Strong gel (EF) & 978 & $8.8 \times 10^{3}$ & 6 \\
\hline 2 & $10^{3}$ & 12 & Weak gel (FF) & 2893 & $3.2 \times 10^{2}$ & 6 \\
\hline 2 & $10^{4}$ & 10 & Repulsive glass & 550 & $3.6 \times 10^{3}$ & 2(b), 4(a), 5, 9(b) \\
\hline 2 & $10^{4}$ & 8.5 & Strong gel (EF) & 825 & $7.0 \times 10^{3}$ & $4(\mathrm{~b}), 5,8(\mathrm{c}), 9(\mathrm{c})$ \\
\hline 2 & $10^{4}$ & 12 & Weak gel (FF) & 1520 & $1.5 \times 10^{2}$ & $4(\mathrm{c}), 5,8(\mathrm{c}), 9(\mathrm{c})$ \\
\hline 3 & 0 & 10 & Repulsive glass & 735 & $5.9 \times 10^{3}$ & $2(\mathrm{~b}), 8(\mathrm{a}), 9(\mathrm{a})$ \\
\hline 3 & $10^{2}$ & 10 & Strong gel (EF) & 1933 & $2 \times 10^{2}$ & 2(b) \\
\hline 3 & $10^{3}$ & 10 & Weak gel (EF) & 1110 & $1 \times 10^{2}$ & $2(\mathrm{~b})$, \\
\hline 3 & $10^{4}$ & 10 & Weak gel (EF) & 835 & $7.1 \times 10^{3}$ & $2(b), 5,8(b), 10$ \\
\hline
\end{tabular}


$2^{\circ}$, gap height at the tip of cone plate geometry $\left.47 \mu \mathrm{m}\right)$. The storage $\left(G^{\prime}\right)$ and loss $\left(G^{\prime \prime}\right)$ moduli were determined by varying the shear stress amplitude from 1 to $100 \mathrm{~Pa}$ at a constant frequency of $6 \mathrm{rad} / \mathrm{s}$. In preliminary tests, we performed oscillatory shear amplitude sweeps in the range of $1100 \mathrm{~Pa}$ using plate-plate geometry with varying gap height, and markers at the rim were used for visual inspection of sample deformation using video imaging. No indication of the occurrence of slip during such experiments was found.

\section{E. Frequency sweep experiments}

Oscillatory shear flow experiments at varying frequencies were performed in the linear viscoelastic regime, where $G^{\prime}$ and $G^{\prime \prime}$ are independent of the applied stress amplitude. The experiments were carried out using a Physica Anton Paar (MCR501) controlled stress rheometer, with cone-plate geometry (diameter $25 \mathrm{~mm}$, cone angle $2^{\circ}$, gap height at the tip of cone plate geometry $47 \mu \mathrm{m})$. The experiments were executed at a constant deformation amplitude of $1 \%$ from high to low frequency (60 $0.006 \mathrm{rad} / \mathrm{s}$ ). Preliminary amplitude sweep experiments confirmed that this deformation is within the linear viscoelastic response regime for the suspensions investigated here.

\section{F. Oscillatory squeeze flow}

The dependence of $G^{\prime}$ and $G^{\prime \prime}$ on angular frequency $\omega=2 \pi \mathrm{f}$ between $10^{1}$ and $3 \times 10^{4} \mathrm{rad} / \mathrm{s}$ was measured by squeezing samples at very low deformation $(<0.1 \%$, depending on gap height) using a customized piezo-driven axial vibrator. $G^{\prime}$ and $G^{\prime \prime}$ were calculated from the detected phase shift and voltage amplitude determined in experiments with and without the sample in the measuring cell as described earlier [47]. Sample deformation in these experiments was so small that the resulting data were always in the linear viscoelastic regime [47]. The gap height was adjusted to $10100 \mu \mathrm{m}$ depending on the sample composition using appropriate distance rings. The exact gap width was determined from preliminary calibration with a Newtonian fluid of known viscosity. The required sample volume is approximately $100 \mu \mathrm{l}$. To make an accurate comparison, samples aged for 10 weeks were used for these experiments and for the frequency sweep and DWS experiments.

\section{G. DWS}

DWS is an optical microrheological technique based on the thermal motion of embedded tracer particles and covers the angular frequency range from 1 to $10^{6} \mathrm{rad} / \mathrm{s}$. We used $\mathrm{TiO}_{2}$ nanoparticles (LS Instruments AG, Fribourg, Switzerland) of $0.36 \mu \mathrm{m}$ in diameter as tracers; $0.5 \mathrm{wt}$. $\%$ of $\mathrm{TiO}_{2}$ particles were added during preparation of Laponite dispersions, and $800 \mu \mathrm{l}$ samples were filled into cuvettes (Hellma, Müllheim, Germany) with $2 \mathrm{~mm}$ thickness. In accordance with Bonn et al. [34], we observed that the addition of $\mathrm{TiO}_{2}$ particles does not affect the linear viscoelastic response of dispersions. Measurements were conducted and analyzed using a DWS ResearchLab (LS Instruments, Fribourg, Switzerland) with a multitau correlator at an acquisition time of $270 \mathrm{~s}$ and echo duration of $5 \mathrm{~s}$. The mean square displacement (MSD) of tracer particles was calculated from the autocorrelation of the scattered light intensity, and a generalized Stokes-Einstein equation was used to determine the complex shear modulus $G^{*}=G^{\prime}+i G^{\prime \prime}$ [48]. The MSD was calculated for lag times between $10^{1}$ and $10^{6} \mathrm{~s}$, and data analysis was performed as described by Oelschlaeger et al. [49].

\section{H. MPT}

To perform MPT video microscopy, 0.01 vol. \% of dragon green fluorescent polystyrene spheres of either 1.01, 0.52, 0.21 , or $0.19 \mu \mathrm{m}$ in diameter (Bang Laboratories, USA) were added to the Laponite dispersions directly after sample preparation. According to the manufacturer, tracer particles are negatively charged due to initiator sulfate groups and adsorbed ionic surfactant. Thus, these particles should not aggregate with the negatively charged clay particles, even at low $p \mathrm{H}$ when edges are positively charged the repulsion between the tracers and the negatively charged face should be dominating. To determine the significance of negatively charged tracer particles and clay mineral interactions, Rich et al. [40] performed solution microcalorimetry measurements using an Isothermal titration calorimeter and found that such interactions can be safely neglected. The samples including tracer particles were vortexed and homogenized for $5 \mathrm{~min}$ in an ultrasonic bath. They were then directly injected into a commercial rectangular capillary of $0.1 \mathrm{~mm}$ in thickness and $2 \mathrm{~mm}$ in width (CM Scientific, UK) sealed with UV-curing glue. The microscope (Axio Observer D1, Carl Zeiss) equipped with a Fluar $100 \times$, N.A. 1.3, oil-immersion lens was focused roughly halfway into the sample, and the Brownian motion of tracer particles was monitored in a $127 \times 127 \mu \mathrm{m}$ field of view, at a rate of 30 frames per second (sCMOS camera Zyla $\mathrm{X}$ ) for $1 \mathrm{~min}$. The experimental setup was described in detail by Kowalczyk et al. [50]. For each experiment, approximately 150 particles were tracked simultaneously. After image processing (IPS Visiometrics), displacement of particle centers was monitored using a self-written MATLAB code [50] based on the widely used Crocker and Grier tracking algorithm [51]. A highly elastic tri-block-copolymer gel Pluronic F127 (BASF SE, Ludwigshafen, Germany) has been employed to determine the static error, which sets the upper limit for the storage modulus accessible with a given setup [50] and the lower limit for heterogeneity ratio to be obtained with corresponding tracer particles [52].

\section{RESULTS AND DISCUSSION}

\section{A. Structure formation and initial aging in glass and gel states}

To determine the kinetics of structure formation and initial aging of different arrested states of Laponite dispersions, the change of $G^{\prime}$ and $G^{\prime \prime}$ was measured as a function of time using small amplitude oscillatory shear measurement (stress amplitude $\sigma_{0}=0.2 \mathrm{~Pa}$, frequency $\omega=0.6 \mathrm{rad} / \mathrm{s}$ ). Weak and strong gel and glass structures were obtained by dissolving 3 wt. \% Laponite in deionized water and aqueous $\mathrm{NaCl}$ solutions with varying salt concentration up to $10^{2} \mathrm{M} \mathrm{NaCl}$; see Fig. 2(a). Due to the high energy input during transfer of the sample into the rheometer fixture and during the initial 
steady shear period, samples were in the liquid or so-called sol-state when the oscillatory shear experiments started, as confirmed by visual inspection. All samples except the one with $10^{2} \mathrm{M} \mathrm{NaCl}$ remained in this sol-state characterized by a low $G^{\prime \prime}$ value until both moduli increased and finally $G^{\prime}$ exceeded $G^{\prime \prime}$. The characteristic point where $G^{\prime}=G^{\prime \prime}$ is generally assumed to mark the formation of a percolating network [53], and the corresponding time $t_{\mathrm{c}}$ is called the crossover or gelation time. Here, we attribute it to the formation of a sample spanning the arrested state, i.e., the repulsive glass and weak gel [Fig. 2(a)]. The situation is different for the sample including $10^{2} \mathrm{M} \mathrm{NaCl}$. In this case, even the first modulus values accessible after approximately $50 \mathrm{~s}$ (corresponding to five oscillations) are orders of magnitude higher than in the sol-state with $G^{\prime}>G^{\prime \prime}$ and in the subsequent time interval of approximately $10^{3} \mathrm{~min} G^{\prime \prime}$ remain essentially constant, whereas $G^{\prime}$ further increases without reaching a steady state [Fig. 2(a)], as expected [36]. This demonstrates again that a sample spanning the arrested state is formed, and this attractive gel-state forms orders of magnitude more quickly than the glass state. The characteristic crossover time or gelation time $t_{c}$ in this latter case is clearly below $1 \mathrm{~min}$ and has been extrapolated here by fitting straight lines to the first three data points of the $G^{\prime}$ and $G^{\prime \prime}$ curves [Fig. 2(a)]. Accordingly, we estimate $t_{c} \approx 0.25 \mathrm{~min}$ for the strong gel formation compared to $t_{c} \approx 68 \mathrm{~min}$ and $t_{c}$ $\approx 400 \mathrm{~min}$ for the weak gel and glass formations, respectively. Similar experiments have been performed for other clay mineral contents. As expected, arrested states generally form much more quickly at higher clay mineral content, and for both clay mineral contents, the strong gel forms much more quickly than the glass state [Fig. 2(b)]. We attribute this to the strong attractive interactions dominating at high ionic strength. The formation of so-called weak gels at intermediate electrolyte concentration occurs approximately one order of magnitude more quickly than the glass formation. We attribute this to the weaker electrostatic repulsion among 2:1 layers compared to the glass forming low ionic strength suspensions.

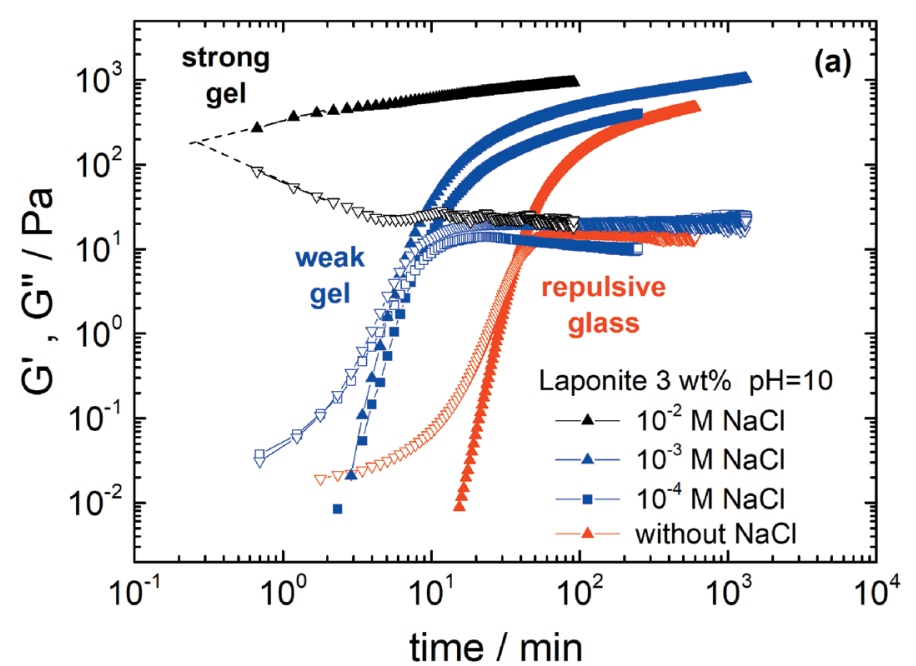

We distinguish between weak and strong gels with reference to $t_{c}$ and ionic strength: Dispersions with intermediate $t_{c}$ (corresponding to intermediate ionic strength) are called weak gels, and finally samples with high ionic strength and distinctly shorter $t_{c}$ are called strong gels.

\section{B. Effect of $p H$ on structure formation and aging}

We applied time-dependent oscillatory shear measurements to Laponite dispersions at $p \mathrm{H}=8.5,10$, and 12 to see the $p \mathrm{H}$ effect on structure formation and aging. Two different Laponite suspensions have been investigated: $2 \mathrm{wt}$. \% solid content without added electrolyte and $1 \mathrm{wt}$. \% solid content with $10^{2} \mathrm{M} \mathrm{NaCl}$. For all investigated samples, a transition to an arrested state with $G^{\prime} \gg G^{\prime \prime}$ is observed. The sample with 2 wt. \% Laponite without added electrolyte at its natural $p \mathrm{H}$ forms a glass, and this takes a long time $\left(t_{c} \approx 1258 \mathrm{~min}\right)$, as discussed above. The arrested states at $p \mathrm{H}=12\left(t_{c}=30 \mathrm{~min}\right)$ and $p \mathrm{H}=8.5\left(t_{c}=0.3 \mathrm{~min}\right)$ form much more quickly (Fig. 3).

A change in $p \mathrm{H}$ of $2 \mathrm{wt} \%$ Laponite dispersion without added $\mathrm{NaCl}$ from its inherent value of $p \mathrm{H}=10$ to $p \mathrm{H}=8.5$ or $p \mathrm{H}=12$ results in a strong increase in electrolyte concentration in the aqueous phase, and the electrical conductivity increases from $505 \mu \mathrm{S} / \mathrm{cm}$ to $892 \mu \mathrm{S} / \mathrm{cm}$ and $1869 \mu \mathrm{S} / \mathrm{cm}$ at $p \mathrm{H}=8.5$ and $p \mathrm{H}=12$, respectively. This leads to a substantial compression of the electrical double layer from $5.5 \mathrm{~nm}$ at $p \mathrm{H}=10$ to $3.4 \mathrm{~nm}$ at $p \mathrm{H}=8.5$ and $2.2 \mathrm{~nm}$ at $p \mathrm{H}=12$, calculated according to Eq. (1). This compression results in the formation of attractive gel states. Moreover, it has to be considered that $p \mathrm{H}_{\mathrm{PZC}}$, edge of Laponite is approximately 1011 as deduced from stability ratio measurements [12] and confirmed by our own CEC measurements according to Meier and Kahr [42] in [45]. These results show a strong increase in $\mathrm{CEC}$ at $p \mathrm{H}>10$ that indicated deprotonation of $(\mathrm{Mg}-\mathrm{OH}-$ $\mathrm{Mg}$ ) surface sites [54] and a strong increase of negative charges at the edges [45].

At $p \mathrm{H}=8.5$, the edges are positively charged, and at $p \mathrm{H}=12$, they are negatively charged. Accordingly, we

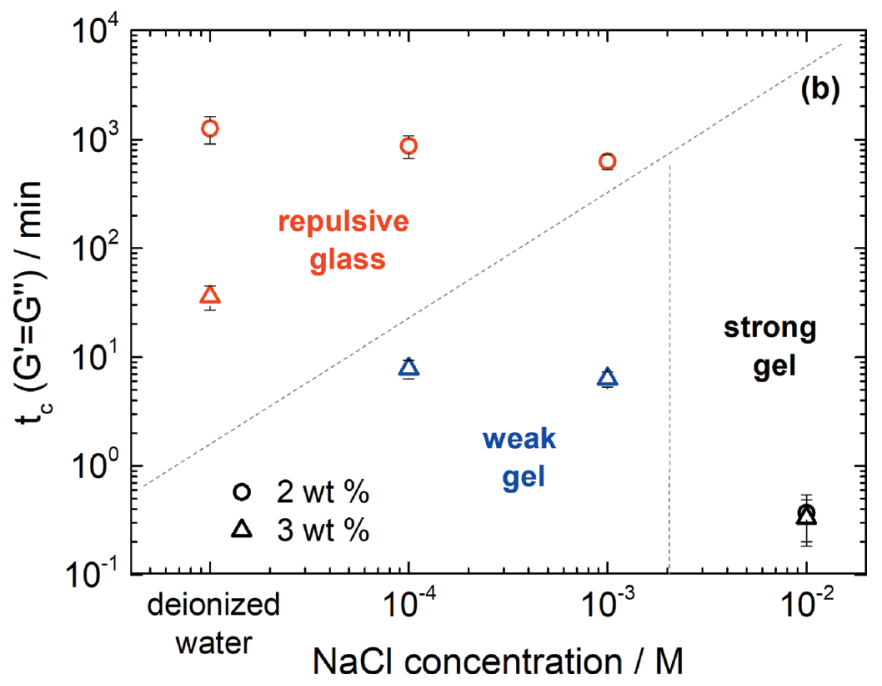

FIG. 2. (a) Structure formation for 3 wt. $\%$ Laponite dispersions at $p \mathrm{H} \quad 10$ with different $\mathrm{NaCl}$ concentration. Solid symbols $\quad G^{\prime}$, open symbols 

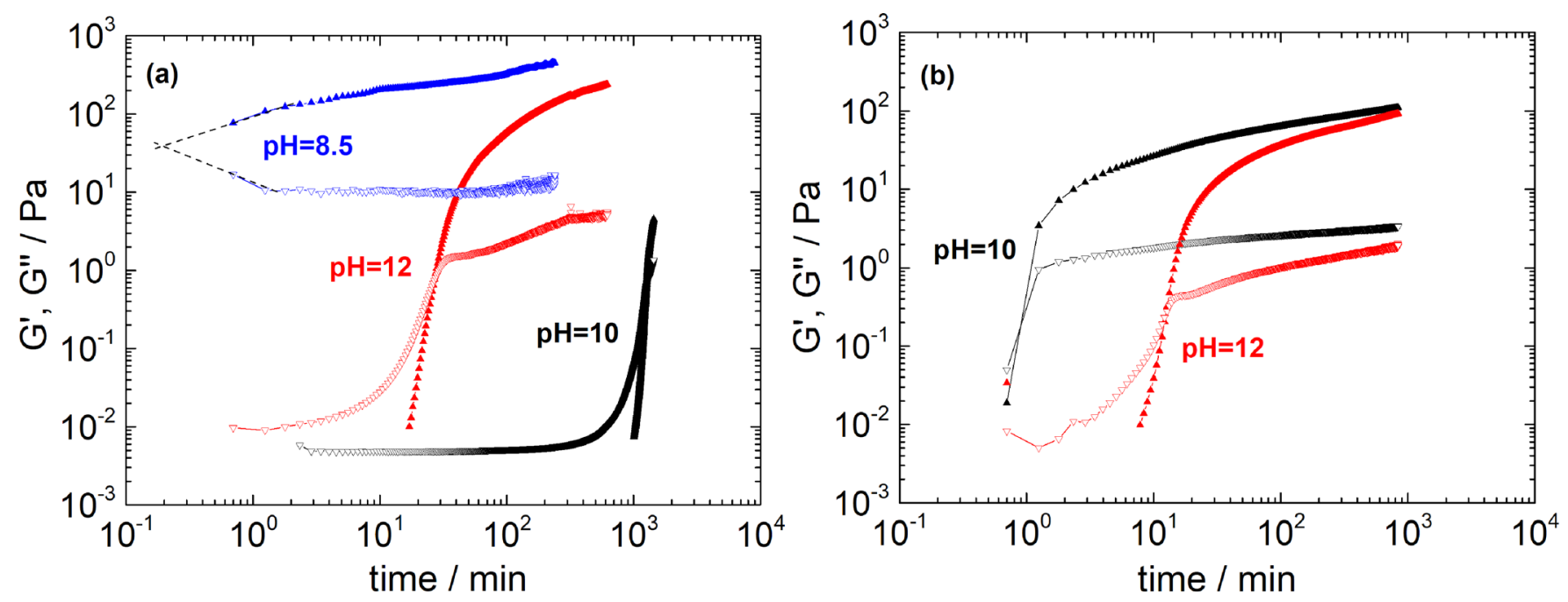

FIG. 3. Effect of $p \mathrm{H}$ on kinetics of structure formation for (a) 2 wt. \% Laponite dispersion without $\mathrm{NaCl}$ and (b) 1 wt. $\% \mathrm{Laponite}$ dispersion at $10{ }^{2} \mathrm{M} \mathrm{NaCl}$ concentration. Solid up triangle $G^{\prime}$, empty down triangle $G^{\prime \prime}$.

assume that EF contacts are likely to form at $p \mathrm{H}=8.5$ and FF contacts with $\mathrm{Na}^{+}$in between prevail at $p \mathrm{H}=12$. Although the electrical double layer around dispersed Laponite layers at $p \mathrm{H}=8.5(\approx 3.4 \mathrm{~nm})$ is larger than at $p \mathrm{H}=12(\approx 2.2 \mathrm{~nm})$, the structure formation is much faster at $p \mathrm{H}=8.5$ than at $p \mathrm{H}=12$ [Fig. 3(a)] because of the oppositely charged edges at $p \mathrm{H}=8.5$. Therefore, we propose that structures predominantly based on EF contacts form much more quickly than FF-dominated structures. This conclusion is further corroborated by results obtained for the 2 and $1 \mathrm{wt}$. $\%$ clay mineral dispersions with $10^{2} \mathrm{M} \mathrm{NaCl}$. Since the kinetics of structure formation for the $2 \mathrm{wt}$. \% Laponite samples are much faster than for the sample with $1 \mathrm{wt} \%$ solid content at high $\mathrm{NaCl}$ concentration, we show the effect of $p \mathrm{H}$ on structure formation here only for the $1 \mathrm{wt} \%$ Laponite dispersions with $10{ }^{2} \mathrm{M} \mathrm{NaCl}$ for the sake of clarity. Due to the high electronic concentration, attractive interactions dominate in both cases at $p \mathrm{H}=10$ and $p \mathrm{H}=12$, and structure formation occurs rapidly [Fig. 3(b)]. At $p \mathrm{H}=10$, edges are still slightly positively charged, and the arrested state is assumed to have an EF structure, whereas FF contacts prevail at $p \mathrm{H}=12$. Again, the structure supposed to be dominated by EF contacts forms more quickly than the gel including FF-type particle contacts.

\section{Aging of Laponite dispersions in different arrested states}

Aging i.e., the change of structure or dynamics over long periods of time is a common phenomenon in out-ofequilibrium systems such as amorphous polymers [55], supersaturated solid solutions [56] or clay mineral dispersions $[31,36,46,57]$. In the latter case, aging corresponds to a perpetual rearrangement of clay mineral layers and reorganization of the overall gel or glass structure and shows up, e.g., in a monotonic increase of the storage modulus over time. This phenomenon can already be seen from the transient $G^{\prime}$ data shown in Figs. 2 and 3 covering a time interval of approximately one day. Willenbacher [36] was the first to discuss aging in attractive gel Laponite dispersions as a self- delaying process and reported an increase of $G^{\prime}$ over several weeks following a power law $G^{\prime} \sim t^{\alpha}$ with $\alpha=0.13 \pm 0.02$ independent of clay mineral content and mechanical pretreatment of samples. Structural rearrangement and aging have been shown to endure for more than one year in dilute Laponite dispersions as revealed by light and X-ray scattering experiments [58]. Here, we have studied the aging phenomenon in different arrested states of Laponite dispersions using shear modulus measurements. We performed fixed frequency amplitude sweep oscillatory shear experiments at time intervals of one week covering a total period of up to 16 weeks. Between measurements, samples were stored in tightly sealed vials to avoid any loss of solvent. Corresponding results for dispersions including $2 \mathrm{wt}$. \% Laponite and $10^{4} \mathrm{M} \mathrm{NaCl}$ at $p \mathrm{H}=8.5,10$, and 12 are shown in Fig. 4. The $p \mathrm{H}$ was periodically tested and confirmed to remain at its initial value during the extended storage time.

The attractive gel formed at $p \mathrm{H}=8.5$ and assumed to have EF particle contacts exhibits the highest strength with a shear modulus $G^{\prime} \approx 10^{3} \mathrm{~Pa}$. This structure also requires the largest critical stress $\sigma_{y}$ to enable flow, usually defined as the stress amplitude at which $G^{\prime}=G^{\prime \prime}$ (often also termed apparent yield stress). Here, this crossover is not visible since catastrophic failure destroys the structure, i.e., $G^{\prime}$ rapidly decays and becomes immeasurably small. The critical stress amplitude at which this occurs is thus termed $\sigma_{y}$ and found to be $\approx 70 \mathrm{~Pa}$. This structure shows very little aging, and the modulus increases by only $10 \%$ within 5 weeks of storage.

The attractive arrested state formed at $p \mathrm{H}=12$ and supposed to have a prevailing FF structure exhibits a much weaker shear strength than the EF structure with an initial modulus $G^{\prime}=10^{2} \mathrm{~Pa}$ and $\sigma_{y}=15 \mathrm{~Pa}$. This type of gel experiences a gradual structural breakdown when it is exceeded and aging is clearly visible, and the modulus increases by a factor of two within 5 weeks. The repulsive glass formed at $p \mathrm{H}=10$ exhibits lower initial shear strength and yield stress than the attractive gel formed at $p \mathrm{H}=12$. However, it exhibits uniquely strong aging, and $G^{\prime}$ increases by a factor of 4 

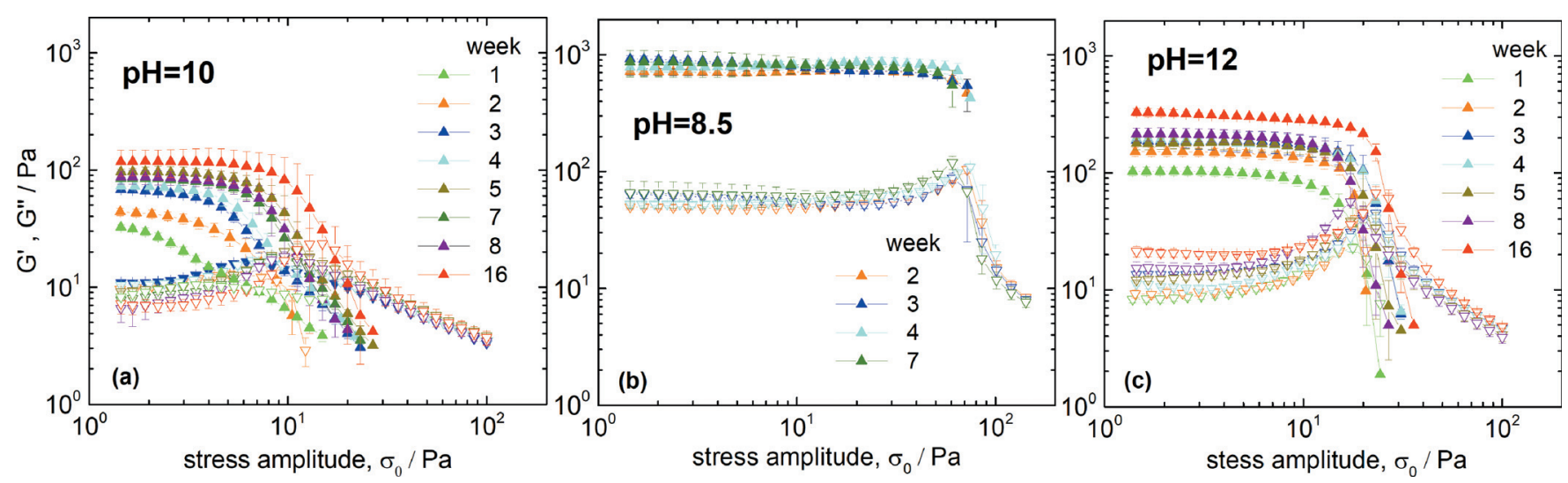

FIG. 4. Amplitude sweep experiments at different aging times for $2 \mathrm{wt}$. \% Laponite dispersions with $10{ }^{4} \mathrm{M}$ added $\mathrm{NaCl}(\mathrm{a})$ at $p \mathrm{H} \quad 10$, (b) at $p \mathrm{H} \quad 8.5$ and (c) at $p \mathrm{H}$ 12. Solid up triangle $G^{\prime}$, open down triangle $G^{\prime \prime}$.

within 16 weeks of storage. Moreover, the mode of failure changes from gradual breakdown for shortly stored samples to catastrophic failure at extended aging times.

Figure 5 compares the time evolution of the normalized storage modulus $G_{\text {norm }}^{\prime}=G^{\prime}\left(t_{\text {storage }}\right) / G^{\prime}\left(t_{\text {storage }}=0\right)$ for different repulsive and attractive arrested states. In all cases, the time dependence of $G^{\prime}$ may be approximated by a power law $G^{\prime} \sim t^{\alpha}$ with $\alpha=0.11 \pm 0.03$ for the attractive strong gel states (circle-symbols) in an excellent agreement with the early findings of Willenbacher [36]. In contrast, the weak gels (triangle-symbols) and repulsive glass (square-symbols) exhibit much stronger aging than strong gels. Here, we found $\alpha=0.74 \pm 0.01$ for the glass with 2 wt. $\%$ Laponite and $10^{4}$ $\mathrm{M} \mathrm{NaCl}$ at $p \mathrm{H}=10$ as well as $\alpha=0.36 \pm 0.01$ for the weak gels. Aging is assumed to be determined by the mobility of individual layers, and apparently layer mobility is much higher for the layers arrested due to electrostatic repulsion of neighboring layers than for those trapped in contact with attractive neighbors in gel structures.

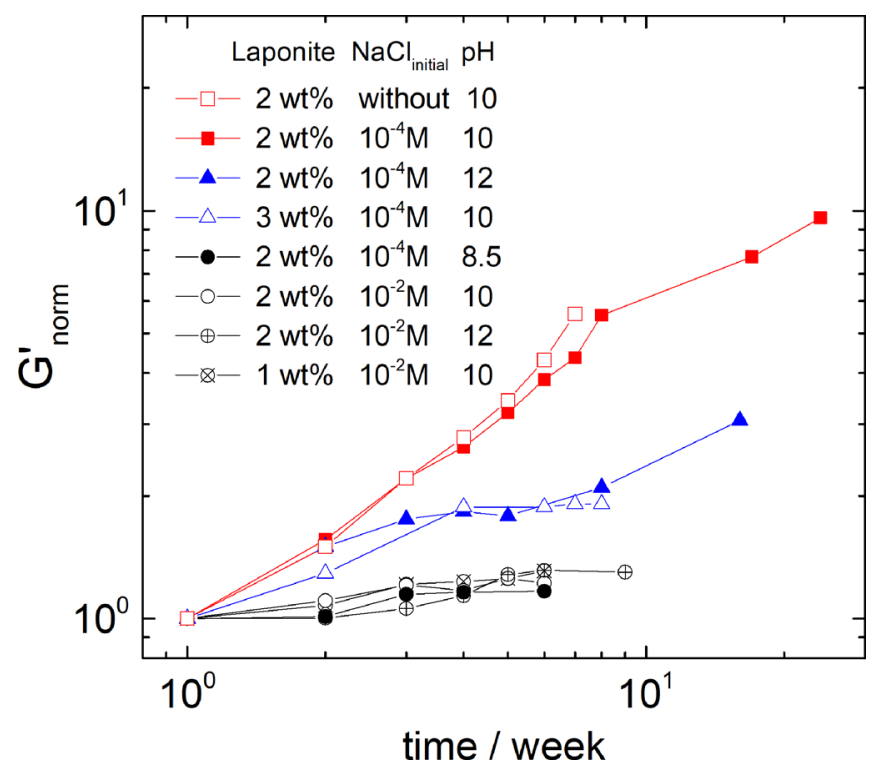

FIG. 5. Normalized plateau moduli as a function of aging time for Laponite dispersions at various solids content and $\mathrm{NaCl}$ concentrations at inherent $p \mathrm{H}$ of 10 , at set $p \mathrm{H} 12$ and 8.5. Circle, triangle, and square symbols represent strong gel, weak gel, and repulsive glass samples, respectively.

\section{Broad bandwidth linear viscoelastic relaxation of Laponite dispersions in different arrested states}

Jabbari-Farouji et al. [33] described the frequency dependence of the shear modulus as a superposition of two power law contributions and found an exponent $\beta \approx 0.7$ characterizing the high frequency relaxation of investigated samples at its inherent $p \mathrm{H}$ and in the frequency range from 6 to $6 \times 10^{5} \mathrm{rad} / \mathrm{s}$. Here, we combined bulk oscillatory shear and squeeze flow with DWS microrheology to cover the frequency range from $5 \times 10^{3}$ to $3 \times 10^{6} \mathrm{rad} / \mathrm{s}$. Storage and loss modulus data for $2 \mathrm{wt}$. \% Laponite dispersions with different $p \mathrm{H}$ and salt concentration are shown in Fig. 6. This extends the work of Jabbari-Farouji et al. [33]. Data obtained with different techniques agree very well, and as expected, $G^{\prime}$ is essentially constant over more than five decades in frequency but starts to increase at approximately $\omega$ $\approx 10^{3} 10^{4} \mathrm{rad} / \mathrm{s}$ [Fig. 6(a)]. The absolute values of the frequency-independent $G^{\prime}$ data in the low frequency range demonstrate that at a given Laponite content. The EF-type attractive gel assumed to be formed at $p \mathrm{H}=8.5$ is stronger than the FF-type arrested state supposed to exist at $p \mathrm{H}=12$, which in turn has a higher shear strength than the repulsive glass $(p \mathrm{H} \mathrm{10).} \mathrm{At} p \mathrm{H} 10$ and 12, adding $\mathrm{NaCl}$ results in an increase of $G^{\prime}$, whereas added electrolyte has no effect at $p \mathrm{H}=8.5$.

At frequencies of approximately $10^{3}$ to $10^{4} \mathrm{rad} / \mathrm{s} G^{\prime \prime}$ also starts to increase strongly according to a power law $G^{\prime \prime} \sim \omega^{\beta}$ with $\beta=0.75 \pm 0.03$ [Fig. 6(b)]. This exponent and the absolute values of $G^{\prime \prime}$ are independent of $p \mathrm{H}$ or salt concentration within experimental uncertainty. Such a scaling law has been predicted for semiflexible chains such as polymers, wormlike micelles or protein filaments $[59,60]$. It is attributed to intrinsic relaxation modes of the building blocks of the semiflexible objects and is directly related to the bending stiffness or persistence length of these chains. Accordingly, highfrequency rheology has been used to study the bending stiffness or persistence length of wormlike micelles [49] and protein [61] solutions, which is related to the bending stiffness of the respective chain building blocks, systematically. Here, this characteristic power law may be related to the bending modulus of the single clay mineral layers, which is independent of the mode of arrest in which the layers are trapped. 

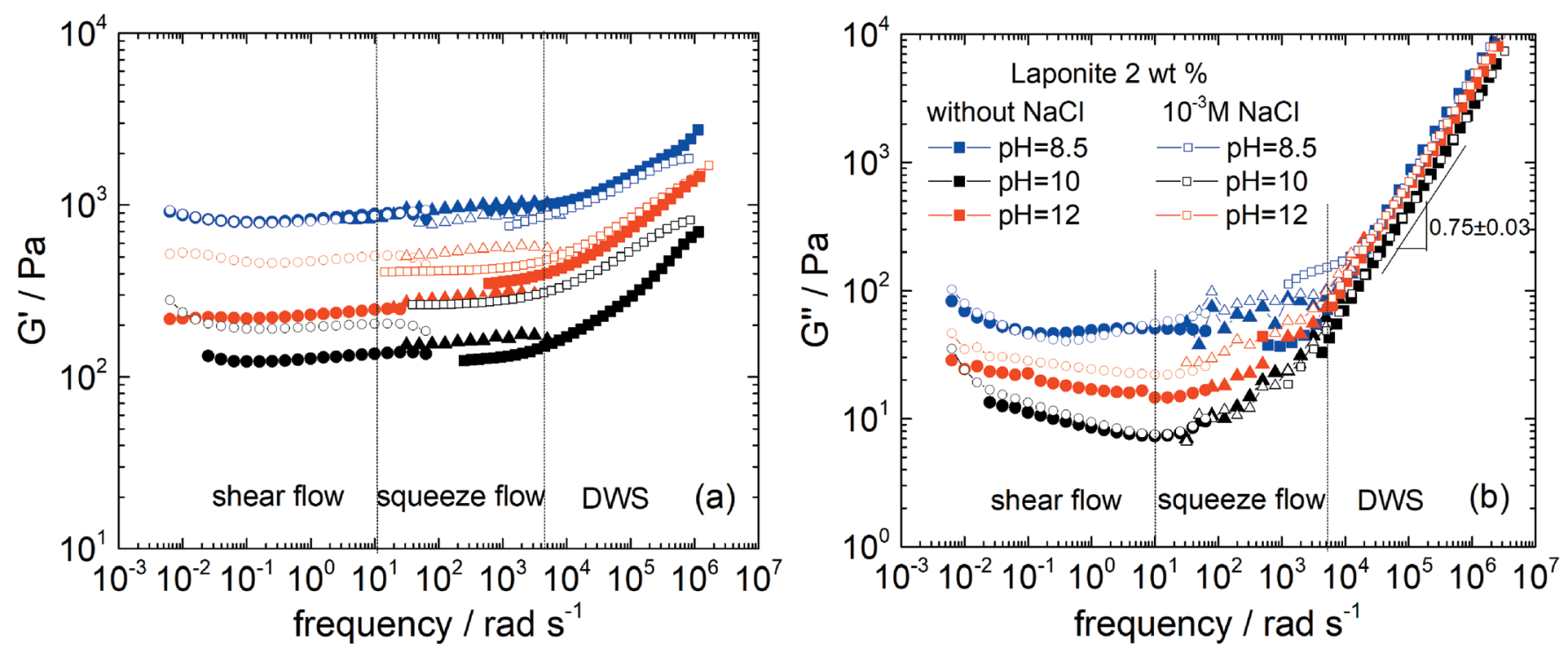

FIG. 6. Linear viscoelastic (a) storage modulus and (b) loss modulus for 2 wt. \% Laponite dispersions with and without $\mathrm{NaCl}$ at three $p \mathrm{H}$ values. The broad fre quency range was covered with the help of small amplitude oscillatory shear flow (square), squeeze flow (triangle), and DWS experiments (square). All experi ments were performed on 10 week old samples.

\section{E. Microrheology and microstructural heterogeneity}

Brownian motion of spherically shaped fluorescent tracer particles embedded in the clay mineral dispersions has been monitored to characterize the change in viscoelasticity and local particle dynamics during the formation of different arrested states. At least 150 tracer particles were tracked simultaneously in a single experiment. From the particle positions in subsequent video images, the mean square displacement $\operatorname{MSD}(\tau)$ of each particle is obtained as a function of lag time $\tau$, and finally the average $\operatorname{MSD}(\tau)$ obtained in a single experiment is calculated.

In Fig. 7(a), such average MSD data obtained at different time intervals after sample preparation are shown for a $3 \mathrm{wt}$. $\%$ Laponite dispersion with $10{ }^{4} \mathrm{M} \mathrm{NaCl}$. Immediately after sample preparation, the MSD of the tracer particles depends linearly on $\tau$, i.e., the tracers diffuse in a viscous environment, the sol state. The slope of the $\operatorname{MSD}(\tau)$ curves then gradually decreases with increasing waiting time until finally MSD data turn time-independent when the arrested gel state is reached.

The Laplace transform of the average $\operatorname{MSD}\left\langle\Delta \tilde{r}^{2}(i \omega)\right\rangle$ is related to the complex shear modulus $G^{*}$ of the surrounding medium via the generalized Stokes-Einstein equation

$$
G^{*}(\omega)=G^{\prime}(\omega)+i G^{\prime \prime}(\omega)=\frac{2 k_{b} T}{\pi d i \omega\left\langle\tilde{\mathbf{r}}^{2}(i \omega)\right\rangle},
$$

where $T$ is the temperature, $k_{b}$ is the Boltzmann constant, and $d$ is the diameter of the tracer particles. This relationship has been used to calculate $G^{\prime}$ and $G^{\prime \prime}$ data from the MSD [50]. The transition from the liquid to the arrested state is clearly visible also from this representation of experimental data [Fig. 7(b)]. Similar results have been reported earlier $[32,40]$, demonstrating that MPT is a versatile, nondestructive way to characterize long-term changes in viscoelastic sample properties even if volatile components are included since the specimens are kept in tightly sealed cuvettes.

Similar experiments have been performed for Laponite dispersions in different arrested states, and structure formation kinetics have been characterized using tracer particles of different size between 0.19 and $1.01 \mu \mathrm{m}$. Formation of the repulsive glass and the attractive gel structure for $3 \mathrm{wt}$. \% Laponite dispersed in deionized water and a $10{ }^{4} \mathrm{M} \mathrm{NaCl}$ solution, respectively, is shown in Figs. 8(a) and 8(b). Structure build-up is characterized via the corresponding change in $G^{\prime}$ and $G^{\prime \prime}$ taken at fixed frequency $\omega=0.6 \mathrm{rad} / \mathrm{s}$ over the waiting time. For comparison, the results from mechanical rheometry are also included.

For the glass sample, the characteristic structure formation time $t_{c}$ at which $G^{\prime}=G^{\prime \prime}$ is independent of the size of the tracer particles, and MPT data are in excellent agreement with bulk rheometry data. This is different from earlier observations [62,63] showing a probe size dependence of tracer diffusivity, but it should be noted that the particle size in these dynamic light scattering and fluorescence recovery after photo bleaching studies is well below the size range investigated here. It should also be noted that the aging of the glasses and gel systems, i.e., the increase in $G^{\prime}$ for times $t>t_{c}$ cannot be monitored using MPT. Due to the constraints defined by the noise-to-signal ratio of our setup, the upper limit for the accessible modulus is $G_{\text {max }}^{\prime} \approx 30 \mathrm{~Pa}$, essentially independent of particle size [50].

A completely different scenario is observed for the sample with 3 wt. \% Laponite and $10{ }^{4} \mathrm{M} \mathrm{NaCl}$ considered as a weak gel. In this case, the crossover times $t_{c}$ from MPT are significantly longer than $t_{c, \text { bulk }}=15$ min obtained from bulk rheometry, i.e., $t_{c}$ increases systematically with decreasing tracer particle size, and for $d=0.21 \mu \mathrm{m} t_{c}=55 \mathrm{~min}$ was found. Similar results were obtained for the sample with 2 wt. \% Laponite and $10^{4} \mathrm{M} \mathrm{NaCl}$; in this case, $t_{c, b u l k}=120 \mathrm{~min}$ and $t_{c}=1224 \mathrm{~min}$ were determined using tracer particles with $d=0.19 \mu \mathrm{m}$. Furthermore, Rich et al. 

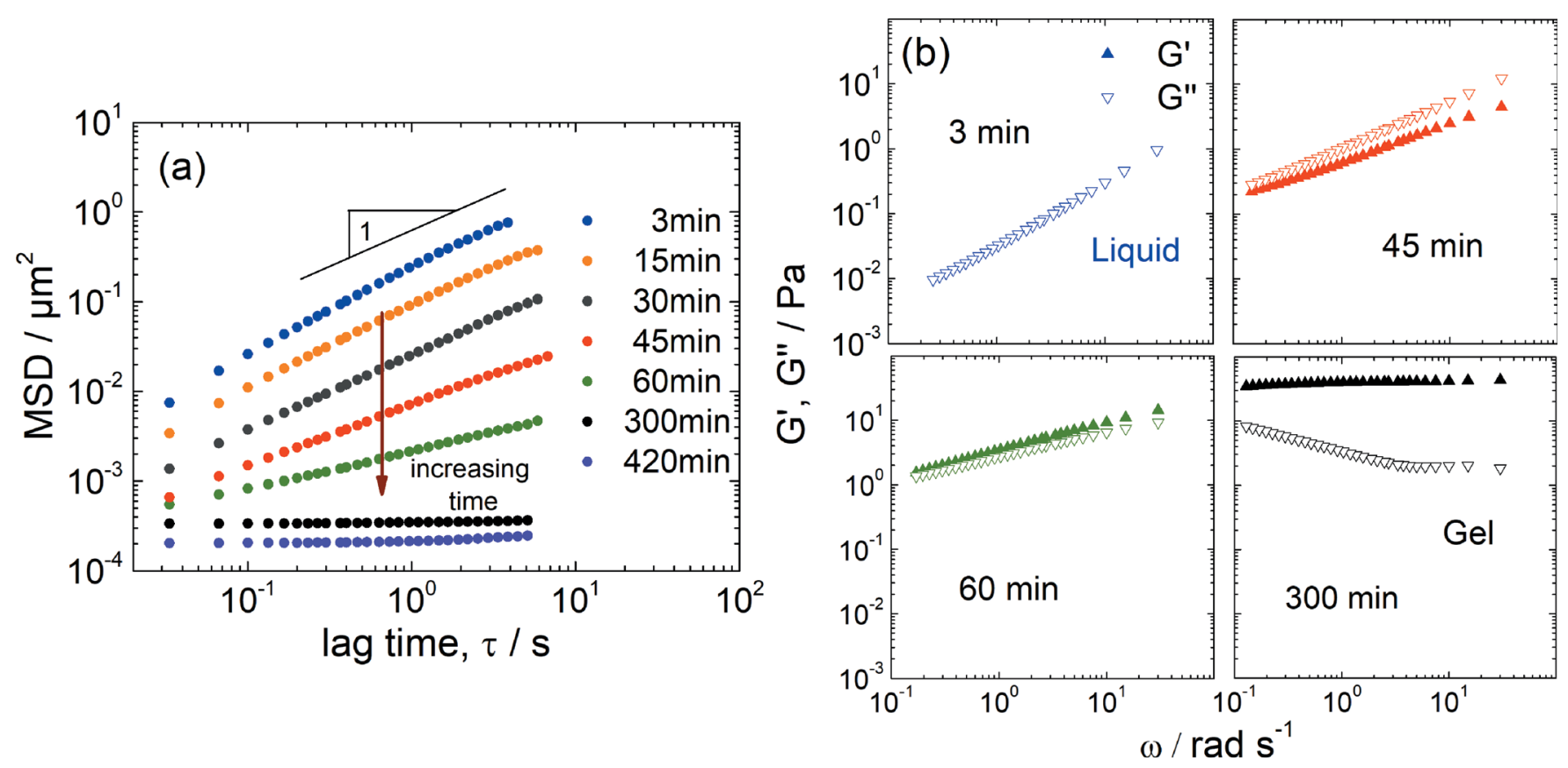

FIG. 7. (a) Mean value of MSD of $0.21 \mu \mathrm{m}$ tracer particles embedded in a 3 wt. \% Laponite dispersion with $10{ }^{4} \mathrm{M} \mathrm{NaCl}$ at $p \mathrm{H} \quad 10$ at different waiting times. (b) Frequency dependence of the storage and loss moduli of $3 \mathrm{wt} \% \%$ Laponite dispersion with $10{ }^{4} \mathrm{M} \mathrm{NaCl}$ on the corresponding length scale (0.21 $\mu$ m) at dif ferent waiting times.

[40] found a shift of $t_{c}$ from $t_{c, b u l k}=6 \mathrm{~min}$ to $t_{c}=180 \mathrm{~min}$ at a Laponite concentration of $1 \mathrm{wt}$. $\%$ using $0.11 \mu \mathrm{m}$ particles, i.e., the delay of the sol-gel transition increases with decreasing clay mineral concentration.

Similar results have been reported previously for Laponite dispersions with solids content between 0.75 and $1.25 \mathrm{wt} . \%$ at $\mathrm{NaCl}$ concentrations of $1.8 \times 10^{3} \mathrm{M}$ and $1 \times 10^{3} \mathrm{M}$, respectively $[32,40]$, which may also be considered as weak gels. This increase in gelation time with decreasing length scale seems to be consistent with the fractal network model proposed by Pignon et al. [64], if layer rearrangements and reorganization of micron-sized clusters assumed to build up the fractal network are considered to occur over long times. On the macroscopic scale, it shows up as slow aging, i.e., weak increase in $G^{\prime}$ over time as discussed above.

Finally, we have compared macro- and microstructure formation for Laponite gels at $p \mathrm{H}=8.5$ where $\mathrm{EF}$ layer contacts prevail and at $p \mathrm{H}=12$ where $\mathrm{FF}$ is the preferred layer contact mode. As seen in Fig. 8(c), in both cases, gelation is
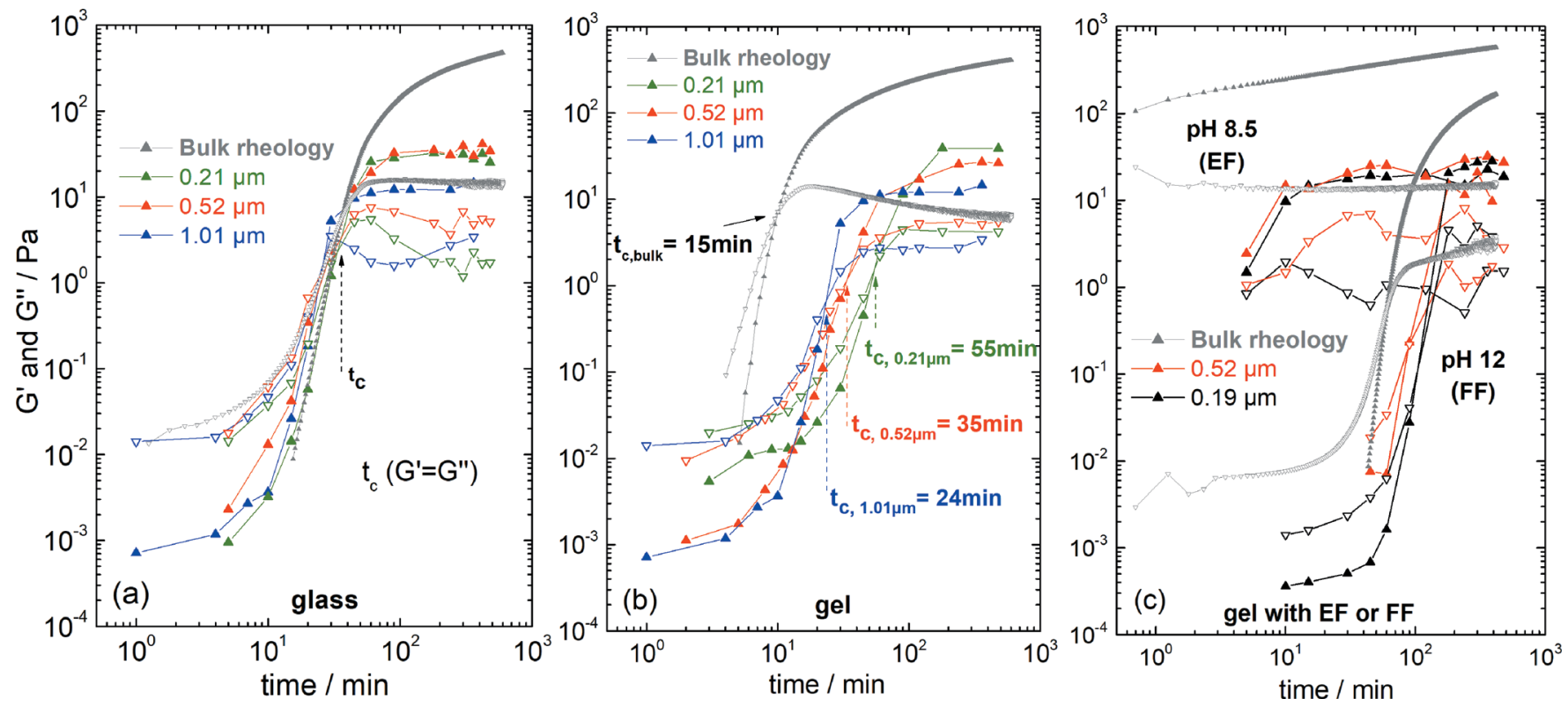

FIG. 8. (a) Glass structure; viscoelastic moduli as a function of time for $3 \mathrm{wt}$ \% Laponite in deionized water at $p \mathrm{H} \quad 10$. (b) Gel structure; viscoelastic moduli as a function of time for 3 wt. \% Laponite in $10{ }^{4} \mathrm{M} \mathrm{NaCl}$ at $p \mathrm{H} \quad 10$. (c) Gel structure with prevailing EF or FF; viscoelastic moduli as a function of time for 2 wt. \% Laponite in $10{ }^{4} \mathrm{M} \mathrm{NaCl}$ aqueous solution at $p \mathrm{H} \quad 8.5(\mathrm{EF})$ and 12 (FF). $G^{\prime}$ (solid up triangle) and $G^{\prime \prime}$ (open down triangle). 
significantly delayed at the microscale compared to the macroscale, and structural refinement even occurs in the strong gel obtained at $p \mathrm{H}=8.5$.

The delayed gelation time observed on the microscale and the corresponding structural refinement seem to indicate a heterogeneity of the attractive gel arrested states, which is not observed in glass systems. MPT is a valuable tool to characterize sample heterogeneities at a submicrometer length scale offering good statistical significance. Oppong et al. [32] quantified sample heterogeneity using the non-Gaussian parameter characterizing the deviation of the MSD distribution at a fixed lag time for the ensemble of tracked particles from the Gaussian distribution function to be found in a homogeneous, ergodic system. For the weak gel, they found that this parameter increased sharply until the (microscopic) gelation time was reached and seemed to remain high even at somewhat longer aging times. Similar results were reported by Rich et al. [40] using the so-called heterogeneity ratio $H R$ as a quantitative measure of spatial heterogeneity obtained by MPT experiments. $H R$ is calculated as [65]

$$
H R=\frac{M_{2}(\tau)}{M_{1}(\tau)^{2}},
$$

where $M_{l}(\tau)$ is the estimator for the ensemble average of MSD, and $M_{2}(\tau)$ is the estimator for the ensemble variance of MSD; both quantities are calculated at a given lag time $\tau$ from individual particle trajectories weighted by a factor proportional to their length. For the weak gel, a monotonic increase of $H R$ with waiting time was observed even somewhat beyond the gelation point obtained at the length scale of the respective tracer particles [40].
Here, we have investigated the heterogeneity of Laponite dispersions with varying clay mineral content and electrolyte concentrations as well as different $p \mathrm{H}(8.5,10$, and 12), thus including glass and different gel arrested states. We used the parameter $H R$ [Eq. (3)] to quantify sample heterogeneity based on MPT experiments with different particle size $(d=0.19,0.21$, and $0.52 \mu \mathrm{m})$. Figure 9 displays these $H R$ data as a function of waiting time $t$, and the latter is normalized to the microscopic gelation time $t_{c}$ determined for each sample using the respective tracer particles.

Note that nonzero $H R$ values are found even for ideal homogeneous fluids due to limitations of experimental setup and data acquisition [65]. This $H R$ threshold has been determined experimentally using a homogenous, highly elastic polymer gel (Puronic F127, $G^{\prime}=10^{4} \mathrm{~Pa}$ at $T=20^{\circ} \mathrm{C}$ ), with corresponding horizontal lines for the different particle sizes (Fig. 9). Experimental data for $t / t_{c} \gg 1$ are close to that threshold for the 0.21 and $0.19 \mu \mathrm{m}$ particles. For the $0.52 \mu \mathrm{m}$ particles, the experimental data are significantly lower; the source of this systematic error could not be resolved.

In these MPT experiments, all investigated samples exhibit a pronounced heterogeneity at the sol-gel/glass transition, i.e., at approximately $t / t_{c} \approx 1$. Consistent with earlier results [33], the glass samples exhibit this heterogeneity only at the length scale of tracer particle size of $0.21 \mu \mathrm{m}$ but not at larger scales. The degree of heterogeneity seems to be most pronounced for the gel with the EF structure generated at $p \mathrm{H}=8.5$ and weakest for the gels formed at $p \mathrm{H}=10$. It should be noted that the HR values are plotted versus $t / t_{c}$ with $t_{c}$ obtained for the used tracer particles. This means that heterogeneity is characterized close to the bulk or macroscopic transition to the arrested state for the glass (see Fig. 8) and the FF-type gel at $p \mathrm{H}=12$. In contrast, the MPT results
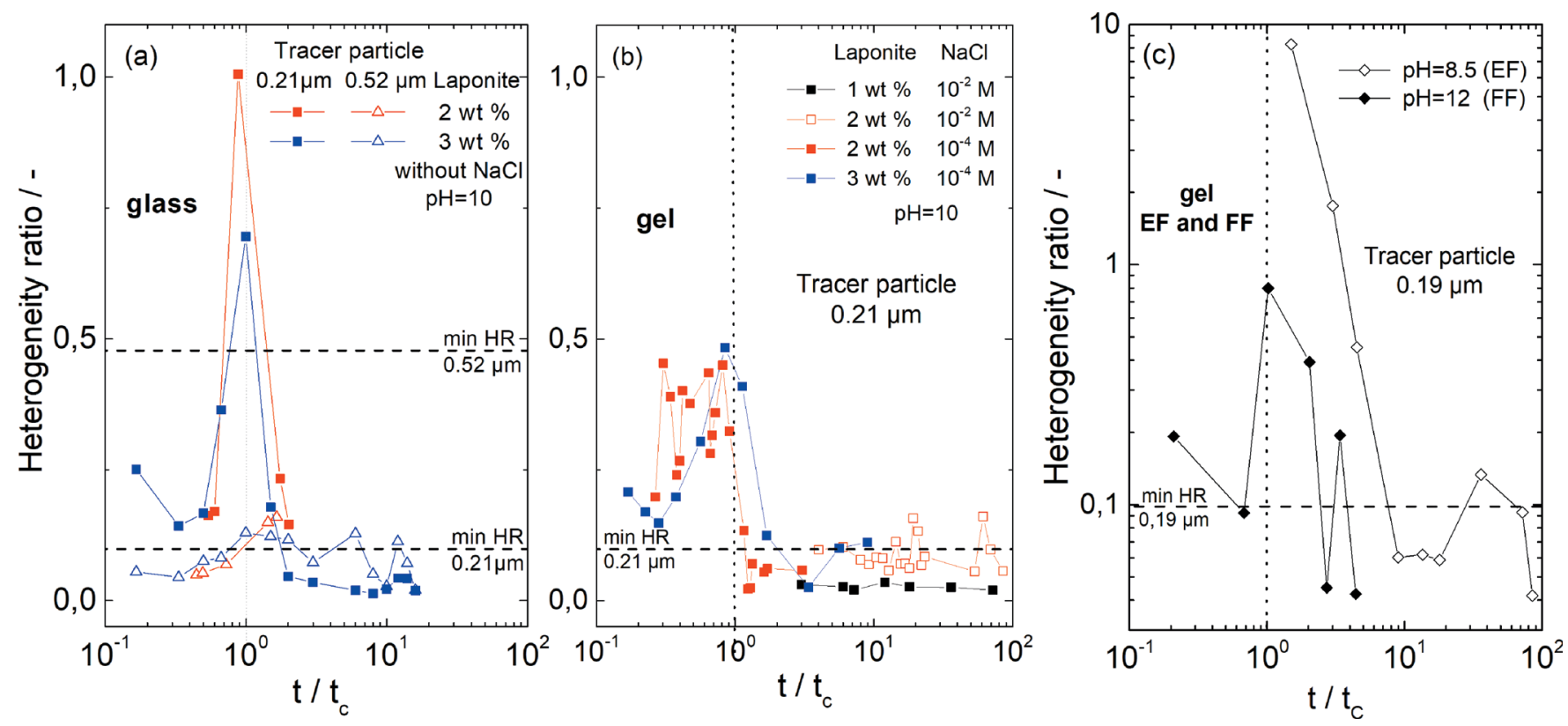

FIG. 9. (a) Heterogeneity ratio (HR) during sol glass phase transition of Laponite dispersions at $p \mathrm{H} \quad 10$ measured by the MPT method and using 0.21 and $0.52 \mu \mathrm{m}$ tracer particles (b) HR during sol gel phase transition of Laponite dispersions at $p \mathrm{H} \quad 10$ measured by MPT method and using $0.21 \mu \mathrm{m}$ tracer particle (c) HR during phase transition of 2 wt. \% Laponite in $10{ }^{4} \mathrm{M} \mathrm{NaCl}$ aqueous solution at $p \mathrm{H} \quad 8.5$ and 12 measured by MPT method and using $0.19 \mu \mathrm{m}$ tracer particle. The minimal HR for each tracer particle type was determined in homogeneous polymer hydrogel (Pluronic F127) and plotted as a horizontal dashed line. The phase transition point $\mathrm{t}_{\mathrm{c}}$, where $G^{\prime}$ and $G^{\prime \prime}$ are equal, was marked at $\mathrm{t} / \mathrm{t}_{\mathrm{c}} \quad 1$ as a vertical dotted line. 

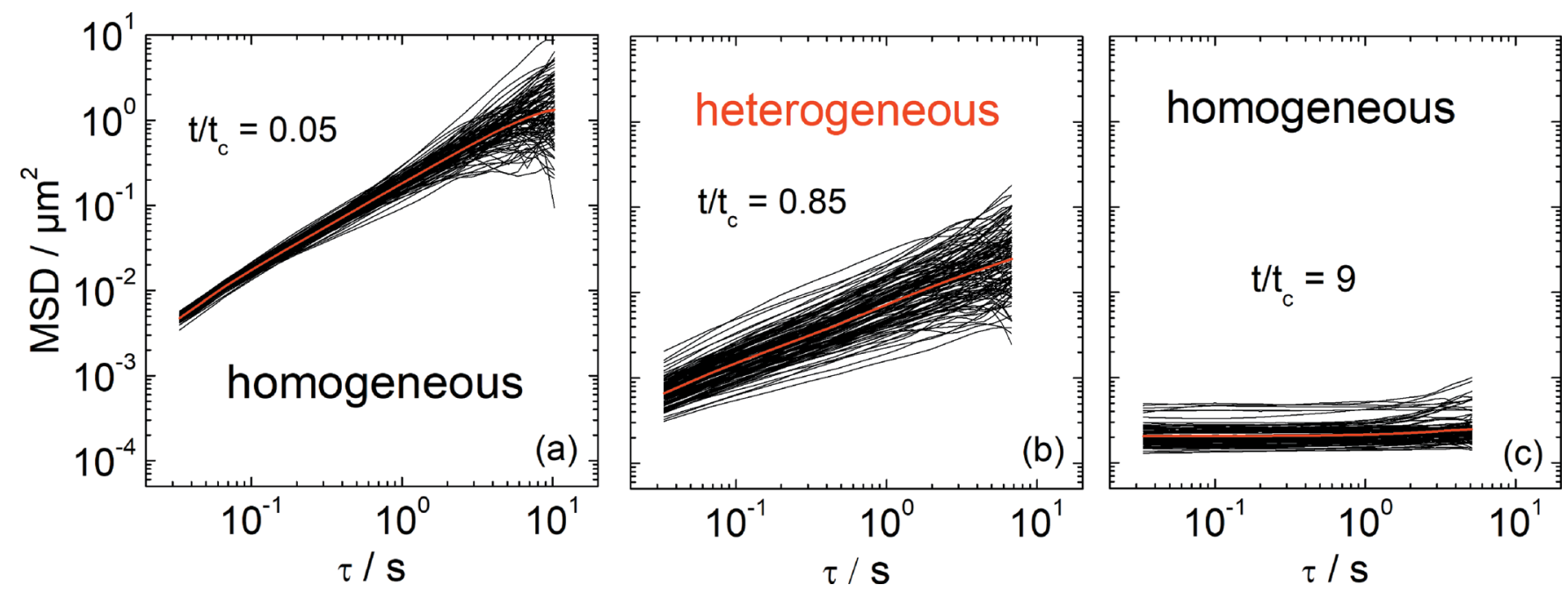

FIG. 10. Ensemble of individual MSD for simultaneously tracked tracer particles (d $0.21 \mu \mathrm{m})$ in a 3 wt. \% Laponite dispersion with $10{ }^{4} \mathrm{M}$ NaCl at different times normalized by crossover time; a broad distribution of MSDs for the heterogeneous structure (b) at $t / t_{c} \approx 1$ and narrow distributions of MSDs for homoge neous structures (a) at $t / t_{c} \ll 1$ and (c) at $t / t_{c} \gg 1$ are clearly visible.

for the weak gels shown in Fig. 9(b) are clearly taken well after the macroscopic gelation occurred, and this might be the reason why the degree of heterogeneity is less pronounced. For the quick gelation system at $p \mathrm{H}=8.5$ [Figs. 8(c) and 9(c)], the structure may even change during ongoing experiments prohibiting straight forward interpretation.

All arrested states exhibit a uniform structure at a length scale of approximately 10 times the clay mineral layer diameter. Undoubtedly, there is no indication of sample heterogeneity for any of the investigated glasses, weak or strong gels at times $t / t_{c} \gg 1$. This surprising result is directly visible from the MSD ensembles exemplary shown in Fig. 10.

The tracer particles to be used for reliable MPT experiments are approximately 10 times larger than the Laponite particles. Hence, it is difficult to resolve structural heterogeneities, e.g., addressed in fundamental work about gelation and phase separation in suspension of spherical particle with short-range attraction [66], and this is supposed to be why all arrested states are seen to be homogeneous at $t \gg t_{c}$.

\section{CONCLUSION}

We have investigated the structure formation and aging of Laponite dispersions in different arrested states. By varying clay mineral content (1 3 wt. \%), $\mathrm{NaCl}$ concentration (up to $\left.10^{2} \mathrm{M} \mathrm{NaCl}\right)$ and $p \mathrm{H}(8.5,10$, and 12) glasses, strong and weak gels were formed. Mechanical oscillatory shear rheometry was used to monitor structure formation and aging. Additional insight into structural refinement and sample heterogeneities on a length scale of $0.21 \mu \mathrm{m}$ was obtained from MPT experiments using tracer particles with diameter $d$ between 0.19 and $1.01 \mu \mathrm{m}$. The high-frequency linear viscoelastic response of arrested dispersions was determined using oscillatory squeeze flow mechanical rheometry and DWS optical microrheology. The formation of arrested states was characterized by the time $t_{c}$ at which $G^{\prime}=G^{\prime \prime}$ at a fixed frequency. For a given clay mineral content, strong attractive gels (high salt concentration) form much more quickly than weak gels (low salt concentration), particularly the repulsive glass state (no added salt). Gels assumed to have preferred EF layer contacts are stronger and are created much more quickly than gels supposed to have prevailing FF contacts. Aging is much more pronounced for glasses than for attractive gels. For all strong gels, $G^{\prime}$ increases only weakly for $t$ $\gg t_{c}$ according to a power law $G^{\prime} \sim t^{\alpha}$ with $\alpha=0.11 \pm 0.03$. For the repulsive glasses with 2 wt. $\%$ Laponite, $\alpha \approx 0.74 \pm 0.03$ is found, and the exponent for the weak gels $(\alpha \approx 0.36 \pm 0.01)$ is in between.

MPT data reveal a structural refinement at the submicrometer length scale during aging, i.e., when the bulk modulus weakly increases, and the characteristic crossover time $t_{c}$ indicating structure formation increases with decreasing size of the tracers probing their viscoelastic environment. No such refinement is found in glass systems. A pronounced structural heterogeneity occurs during formation of attractive gels and repulsive glasses, i.e., at approximately $t \approx t_{c}$; this transient heterogeneity is most pronounced for the gel with the HOC structure formed at $p \mathrm{H}=8.5$. At times $t \gg t_{c}$ all arrested states appear homogenous at the length scale of $0.2 \mu \mathrm{m}$.

Finally, the linear viscoelastic response of all repulsive and attractive arrested states at high frequencies $\left(\omega>10^{4}\right.$ $\mathrm{rad} / \mathrm{s}$ ) exhibits a power law frequency dependence of $G^{\prime \prime}$ $\sim \omega^{0.75}$ attributed to internal bending modes of individual layers, which are independent of the type of layer contacts or structures in which these layers are trapped.

\section{ACKNOWLEDGMENTS}

The authors gratefully acknowledge the German Academic Exchange Service (DAAD Germany) for financial support. The authors would like to thank Rebekka Maria Cseki for aging measurements and Dr. Laure Delavernhe, Dr. Annett Steudel, and Florian Schnetzer for fruitful discussions. 


\section{References}

[1] Jabbari Farouji, S., H. Tanaka, G. H. Wegdam, and D. Bonn, "Multiple nonergodic disordered states in Laponite suspensions: A phase diagram," Phys. Rev. E 78(6), 061405 (2008).

[2] Levitz, P., E. Lecolier, A. Mourchid, A. Delville, and S. Lyonnard, "Liquid solid transition of Laponite suspensions at very low ionic strength: Long range electrostatic stabilisation of anisotropic colloids," Europhys. Lett. 49(5), 672677 (2000).

[3] Tanaka, H., J. Meunier, and D. Bonn, "Nonergodic states of charged colloidal suspensions: Repulsive and attractive glasses and gels," Phys. Rev. E 69, 031404 (2004).

[4] Mourchid, A., E. Lécolier, H. Van Damme, and P. Levitz, "On visco elastic, birefringent, and swelling properties of laponite clay suspen sions: Revisited phase diagram," Langmuir 14(17), 47184723 (1998).

[5] Mongondry, P., J. F. Tassin, and T. Nicolai, "Revised state diagram of Laponite dispersions,” J. Colloid Interface Sci. 283(2), 397405 (2005).

[6] Ruzicka, B., and E. Zaccarelli, "A fresh look at the Laponite phase dia gram,” Soft Matter 7, 12681286 (2011).

[7] Ruzicka, B., L. Zulian, and G. Ruocco, "More on the phase diagram of laponite," Langmuir 22(3), 11061111 (2006).

[8] Fossum, J. O., "Physical phenomena in clays," Phys. A Stat. Mech. Appl. 270(1), 270277 (1999).

[9] Dijkstra, M., J. P. Hansen, and P. A. Madden, "Gelation of a clay col loid suspension,” Phys. Rev. Lett. 75(11), 22362239 (1995).

[10] Nicolai, T., and S. Cocard, "Structure of gels and aggregates of disk like colloids,” Eur. Phys. J. E 5, 221227 (2001).

[11] Mori, Y., K. Togashi, and K. Nakamura, "Colloidal properties of synthetic hectorite clay dispersion measured by dynamic light scattering and small angle X ray scattering," Adv. Powder Technol. 12(1), 4559 (2001).

[12] Tawari, S. L., D. L. Koch, and C. Cohen, "Electrical double layer effects on the Brownian diffusivity and aggregation rate of Laponite clay particles," J. Colloid Interface Sci. 240, 5466 (2001).

[13] Bonn, D., H. Tanaka, G. Wegdam, H. Kellay, and J. Meunier, “Aging of a colloidal 'Wigner' glass,” Europhys. Lett. 45(1), 5257 (1999).

[14] Tombácz, E., and M. Szekeres, "Colloidal behavior of aqueous mont morillonite suspensions: The specific role of $\mathrm{pH}$ in the presence of indifferent electrolytes," Appl. Clay Sci. 27, 7594 (2004).

[15] Lagaly, G., and S. Ziesmer, "Colloid chemistry of clay minerals: The coagulation of montmorillonite dispersions," Adv. Colloid Interface Sci. 100-102, 105128 (2003).

[16] Odriozola, G., M. Romero Bastida, and F. D. J. Guevara Rodríguez, "Brownian dynamics simulations of Laponite colloid suspensions," Phys. Rev. E 70(2), 115 (2004).

[17] Jonsson, B., C. Labbez, and B. Cabane, "Interaction of nanometric clay platelets," Langmuir 24(20), 1140611413 (2008).

[18] Van Olphen, H., An Introduction to Clay Colloid Chemistry, for Clay Technologists, Geologists, and Soil Scientists, 2nd ed. (John Wiley \& Sons, New York, 1977).

[19] Dijkstra, M., J. P. Hansen, and P. A. Madden, "Statistical model for the structure and gelation of smectite clay suspensions," Phys. Rev. E 55(3), 30443053 (1997).

[20] Okamoto, M., P. H. Nam, P. Maiti, T. Kotaka, N. Hasegawa, and A. Usuki, "A house of cards structure in polypropylene/clay nanocompo sites under elongational flow," Nano Lett. 1(6), 295298 (2001).

[21] Hofmann, U., and A. Hausdorf, "Uber das Sedimentvolumen und die Quellung von Bentonit,” Kolloid Zeitschrift 110(1), 117 (1945).

[22] Ramos Tejada, M. M., F. J. Arroyo, R. Perea, and J. D. G. Durán, "Scaling behavior of the rheological properties of montmorillonite sus pensions: Correlation between interparticle interaction and degree of flocculation," J. Colloid Interface Sci. 235(2), 251259 (2001).
[23] Durán, J. D. G., M. M. Ramos Tejada, F. J. Arroyo, and F. González Caballero, "Rheological and electrokinetic properties of sodium montmorillonite suspensions. I. Rheological properties and interparticle energy of interaction,” J. Colloid Interface Sci. 229, 107117 (2000).

[24] Zbik, M. S., D. J. Williams, Y. F. Song, and C. C. Wang, "The forma tion of a structural framework in gelled Wyoming bentonite: Direct observation in aqueous solutions," J. Colloid Interface Sci. 435, 119127 (2014).

[25] Abend, S., and G. Lagaly, "Sol gel transitions of sodium montmoril lonite dispersions," Appl. Clay Sci. 16, 201227 (2000).

[26] Giannakopoulos, E., P. Stathi, K. Dimos, D. Gournis, Y. Sanakis, and Y. Deligiannakis, "Adsorption and radical stabilization of humic acid analogues and $\mathrm{Pb} 2+$ on restricted phyllomorphous clay," Langmuir 22(16), 68636873 (2006).

[27] Ruzicka, B., L. Zulian, and G. Ruocco, "Routes to gelation in a clay suspension," Phys. Rev. Lett. 93(25), 258301 (2004).

[28] Ruzicka, B., L. Zulian, R. Angelini, M. Sztucki, A. Moussard, and G. Ruocco, "Arrested state of clay water suspensions: Gel or glass?," Phys. Rev. E 77(2), 020402 (2008).

[29] Jabbari Farouji, S., R. Zargar, G. H. Wegdam, and D. Bonn, "Dynamical heterogeneity in aging colloidal glasses of Laponite," Soft Matter 8, 55075512 (2012).

[30] Jabbari Farouji, S., G. H. Wegdam, and D. Bonn, "Gels and glasses in a single system: Evidence for an intricate free energy landscape of glassy materials," Phys. Rev. Lett. 99(6), 065701 (2007).

[31] Knaebel, A., M. Bellour, J. P. Munch, V. Viasnoff, F. Lequeux, and J. L. Harden, "Aging behavior of Laponite clay particle suspensions," Europhys. Lett. 52(1), 7379 (2000).

[32] Oppong, F. K., P. Coussot, and J. R. De Bruyn, "Gelation on the microscopic scale,” Phys. Rev. E 78(2), 021405 (2008).

[33] Jabbari Farouji, S., M. Atakhorami, D. Mizuno, E. Eiser, G. H. Wegdam, F. C. MacKintosh, D. Bonn, and C. F. Schmidt, "High band width viscoelastic properties of aging colloidal glasses and gels," Phys. Rev. E 78(6), 061402 (2008).

[34] Bonn, D., S. Tanase, B. Abou, H. Tanaka, and J. Meunier, "Laponite: Aging and shear rejuvenation of a colloidal glass," Phys. Rev. Lett. 89(1), 015701 (2002).

[35] Shahin, A., and Y. M. Joshi, "Irreversible aging dynamics and generic phase behavior of aqueous suspensions of Laponite," Langmuir 26(6), 42194225 (2010).

[36] Willenbacher, N., "Unusual thixotropic properties of aqueous disper sions of Laponite RD," J. Colloid Interface Sci. 182(2), 501510 (1996).

[37] Labanda, J., and J. Llorens, "Effect of aging time on the rheology of Laponite dispersions," Colloids Surf. A Physicochem. Eng. Asp. 329, 16 (2008).

[38] Mourchid, A., A. Delville, J. Lambard, E. Lécolier, and P. Levitz, "Phase diagram of colloidal dispersions of anisotropic charged par ticles: Equilibrium properties, structure, and rheology of Laponite sus pensions," Langmuir 11(6), 19421950 (1995).

[39] Kroon, M., W. L. Vos, and G. H. Wegdam, "Structure and formation of a gel of colloidal disks," Phys. Rev. E 57(2), 19621970 (1998).

[40] Rich, J. P., G. H. McKinley, and P. S. Doyle, "Size dependence of microprobe dynamics during gelation of a discotic colloidal clay," J. Rheol. 55(2), 273299 (2011).

[41] Bonn, D., H. Kellay, H. Tanaka, G. Wegdam, and J. Meunier, "Laponite: What is the difference between a gel and a glass?," Langmuir 15(22), 75347536 (1999).

[42] Meier, L. P., and G. Kahr, "Determination of the cation exchange capacity (CEC) of clay minerals using the complexes of copper(II) ion 
with triethylenetetramine and tetraethylenepentamine," Clays Clay Miner. 47(3), 386388 (1999).

[43] Delavernhe, L., A. Steudel, G. K. Darbha, T. Schafer, R. Schuhmann, C. Woll, H. Geckeis, and K. Emmerich, "Influence of mineralogical and morphological properties on the cation exchange behavior of dio ctahedral smectites," Colloids Surf. A Physicochem. Eng. Asp. 481, 591599 (2015)

[44] Awasthi, V., and Y. M. Joshi, "Effect of temperature on aging and time temperature superposition in nonergodic Laponite suspensions," Soft Matter 5, 49914996 (2009).

[45] Delavernhe, L., M. Pilavtepe, and K. Emmerich, "Cation exchange capacity of natural and synthetic hectorite," Appl. Clay Sci. 151, 175180 (2018).

[46] Au, P. I., S. Hassan, J. Liu, and Y. K. Leong, "Behaviour of Laponite gels: Rheology, ageing, pH effect and phase state in the presence of dispersant," Chem. Eng. Res. Des. 101, 6573 (2015).

[47] Crassous, J. J., R. Re'gisser, M. Ballauff, and N. Willenbacher, "Characterization of the viscoelastic behavior of complex fluids using the piezoelastic axial vibrator," J. Rheol. 49(4), 851863 (2005).

[48] Mason, T. G., and D. A. Weitz, "Optical measurements of frequency dependent linear viscoelastic moduli of complex fluids," Phys. Rev. Lett. 74(7), 12501253 (1995)

[49] Oelschlaeger, C., M. Schopferer, F. Scheffold, and N. Willenbacher, "Linear to branched micelles transition: A rheometry and diffusing wave spectroscopy (DWS) study," Langmuir 25(2), 716723 (2009).

[50] Kowalczyk, A., C. Oelschlaeger, and N. Willenbacher, "Tracking errors in 2D multiple particle tracking microrheology," Meas. Sci. Technol. 26, 015302 (2015).

[51] Crocker, J. C., and D. G. Grier, "Methods of digital video microscopy for colloidal studies,” J. Colloid Interface Sci. 179, 298310 (1996).

[52] Savin, T., and P. S. Doyle, "Static and dynamic errors in particle track ing microrheology,” Biophys. J. 88(1), 623638 (2005)

[53] Winter, H. H., and F. Chambon, "Analysis of linear viscoelasticity of a crosslinking polymer at the gel point," J. Rheol. 30(2), 367382 (1986).
[54] Tournassat, C., E. Ferrage, C. Poinsignon, and L. Charlet, "The titra tion of clay minerals: II. Structure based model and implications for clay reactivity," J. Colloid Interface Sci. 273, 234246 (2004).

[55] Struik, L. C. E., Physical Aging in Amorphous Polymers and Other Materials (Elsevier Scientific Pub. Co., New York, 1978).

[56] Lifshitz, I. M., and V. V. Slyozov, "The kinetics of precipitation from supersaturated solid solutions,” J. Phys. Chem. Solids 19(1), 3550 (1961).

[57] Ianni, F., R. Di Leonardo, S. Gentilini, and G. Ruocco, “Aging after shear rejuvenation in a soft glassy colloidal suspension: Evidence for two different regimes," Phys. Rev. E 75(1), 011408 (2007).

[58] Ruzicka, B., E. Zaccarelli, L. Zulian, R. Angelini, M. Sztucki, A. Moussaid, T. Narayanan, and F. Sciortino, "Observation of empty liquids and equilibrium gels in a colloidal clay," Nat. Mater. 10, 5660 (2011).

[59] Morse, D. C., "Viscoelasticity of tightly entangled solutions of semi flexible polymers," Phys. Rev. E 58(2), R1237 R1240 (1998).

[60] Gittes, F., and F. MacKintosh, "Dynamic shear modulus of a semiflexi ble polymer network," Phys. Rev. E 58(2), R1241 R1244 (1998).

[61] Pawelzyk, P., N. Mucke, H. Herrmann, and N. Willenbacher, "Attractive interactions among intermediate filaments determine net work mechanics in vitro," PLoS One 9(9), 19 (2014).

[62] Petit, L., C. Barentin, J. Colombani, C. Ybert, and L. Bocquet, "Size dependence of tracer diffusion in a laponite colloidal gel," Langmuir 25(20), 1204812055 (2009).

[63] Strachan, D. R., G. C. Kalur, and S. R. Raghavan, "Size dependent diffusion in an aging colloidal glass," Phys. Rev. E 73(4), 041509 (2006).

[64] Pignon, F., A. Magnin, J. M. Piau, B. Cabane, P. Lindner, and O. Diat, "Yield stress thixotropic clay suspension: Investigation of structure by light, neutron, and x ray scattering,” Phys. Rev. E 56(3), 32813289 (1997).

[65] Savin, T., and P. S. Doyle, "Statistical and sampling issues when using multiple particle tracking," Phys. Rev. E 76(2), 021501 (2007).

[66] Lu, P. J., E. Zaccarelli, F. Ciulla, A. B. Schofield, F. Sciortino, and D. A. Weitz, "Gelation of particles with short range attraction," Nature 453, 499503 (2008). 


\section{Repository KITopen}

Dies ist ein Postprint/begutachtetes Manuskript.

Empfohlene Zitierung:

Pilavtepe, M.; Recktenwald, S. M.; Schuhmann, R.; Emmerich, K.; Willenbacher, N. Macro- and microscale structure formation and aging in different arrested states of Laponite dispersions.

2018. Journal of rheology, 62.

doi:10.5445/IR/1000080668

Zitierung der Originalveröffentlichung:

Pilavtepe, M.; Recktenwald, S. M.; Schuhmann, R.; Emmerich, K.; Willenbacher, N. Macro- and microscale structure formation and aging in different arrested states of Laponite dispersions.

2018. Journal of rheology, 62 (2), 593-605.

doi:10.1122/1.5001382 